\title{
DISTORTION AND COEFFICIENT ESTIMATION OF SCHLICHT FUNCTIONS
}

HU KE

ABSTRACT. Let

$$
S(k)=\left\{f(z)=z+\sum_{n-2}^{\infty} a_{n} z^{n} \in S, \varlimsup_{\rho \rightarrow 1^{-}} \frac{(1-\rho)^{2}}{\rho} \max _{|=|-\rho}|f(z)|=\alpha_{f} \geqslant k>0\right\} \text {. }
$$

In this paper, we prove that for $f(z) \in S(k)$ the inequality

$$
\left|a_{n}\right|<n \sqrt{\frac{P+\sqrt{(1+p) A^{1 / 2}-p}}{1+P}}
$$

holds where $p=k^{2} /\left(1-k^{2}\right)$ and $1 \leqslant A<(1.0657)^{\mathrm{x}}$. This strengthens a recent result of Horowitz.

Let $S=\left\{f(z)=z+\sum_{n=2}^{\infty} a_{n} z^{n}: f\right.$ is analytic and univalent in the unit disc $|z|<1\}$ and let $S(k)=\left\{f(z) \in S, \lim _{\rho \rightarrow 1^{-}}(1-\rho)^{2} / \rho \max _{|z|=\rho}|f(z)|=\alpha_{f}>k>\right.$ $0\}$.

(In fact for $f(z) \in S$, the $\lim _{\rho \rightarrow 1^{-}}$exists, but for this work it suffices to use the lim sup.)

The Bieberbach Conjecture asserts that if $f(z)=z+\sum_{n=2}^{\infty} a_{n} z^{n}$ is in $S$, then $\left|a_{n}\right| \leqslant n(n=2,3, \ldots)$. In other words the extremal function of the Bieberbach Conjecture is $z /(1-z)^{2}$ for which $\alpha_{f}=1$. The best approximation to the conjecture so far proved is due to D. Horowitz [1] who showed that

$$
\left|a_{n}\right|<1.0657 n \quad(n \geqslant 2) .
$$

In this paper, we improve Horowitz's result (1). Besides, we prove a new theorem of distortion of schlicht functions. The method of [1] is used.

\section{The main inequalities.}

THEOREM 1. If $f(z)=z+\sum_{n=2}^{\infty} a_{n} z^{n} \in S(k)$, then

$$
\left|a_{n}\right| \leqslant n \sqrt{\frac{P+\sqrt{(1+p) A^{1 / 2}-P}}{1+P}}<c n, \quad n=2,3, \ldots,
$$

where $c=1.0657[1], P=k^{2} /\left(1-k^{2}\right), A=\max \left\{\frac{209}{140}, c^{8}-P\left[\left(\frac{209}{140}\right)^{1 / 2}-\frac{7}{6}\right]^{2}\right\}$.

This result is obviously stronger than (1).

Received by the editors June 13, 1980.

1980 Mathematics Subject Classification. Primary 30C50; Secondary 30C55.

$\mathrm{Key}$ words and phrases. Univalent functions, schlicht functions, coefficients, quadratic inequalities, distortion.

(C)1983 American Mathematical Society $0002-9939 / 82 / 0000-0868 / \$ 02.75$ 
THEOREM 2. If $f(z)=z+\sum_{n=2}^{\infty} a_{n} z^{n} \in S(k)$, then

$$
\begin{aligned}
\left.\left.\left|\sum_{j=1}^{L}\right| a_{j}\right|^{2} x_{j}\right|^{2} & +P\left|\sum_{j=1}^{L}\left(\left|a_{j}\right|^{2}-j^{2}\right) x_{j}\right|^{2} \\
& \leqslant \sum_{m, n=1}^{L} \sum_{K=1}^{n+m-1} \beta_{k}(n, m)\left|a_{k}\right|^{2} x_{m} \bar{x}_{n},
\end{aligned}
$$

where

$$
\beta_{k}(n, m)= \begin{cases}n-|k-m| & \text { for }|k-m|<n, \\ 0 & \text { otherwise }\end{cases}
$$

and $P=k^{2} /\left(1-k^{2}\right)$.

Proof. We know that [2]

$$
\sum_{p . q=0}^{m} y_{p} \bar{y}_{q}\left\{\left|\frac{f_{p}-f_{q}}{z_{p}-z_{q}}\right|^{2} \frac{1}{\left|1-z_{p} \bar{z}_{q}\right|^{2}}-\left|\frac{f_{p} f_{q}}{z_{p} z_{q}}\right|^{2}\right\} \geqslant 0, \quad\left(f_{p}=f\left(z_{p}\right)\right) .
$$

In (1.3), set $y_{p}=\lambda_{p} x_{z}(p \neq 0), y_{0}=x$, and let

$$
\begin{aligned}
& A_{1,2}=\sum_{p=1}^{m} \lambda_{p}\left\{\left|\frac{f_{p}-f_{0}}{z_{p}-z_{0}}\right|^{2} \frac{1}{\left|1-z_{p} \bar{z}_{0}\right|^{2}}-\left|\frac{f_{p} f_{0}}{z_{p} z_{0}}\right|^{2}\right\}, \quad \overline{A_{12}}=A_{21}, \\
& A_{2,2}=\sum_{p, q=1}^{m} \lambda_{p} \bar{\lambda}_{q}\left\{\left|\frac{f_{p}-f_{q}}{z_{p}-z_{q}}\right|^{2} \frac{1}{\left|1-z_{p} \bar{z}_{q}\right|^{2}}-\left|\frac{f_{p} f_{q}}{z_{p} z_{q}}\right|^{2}\right\}, \\
& A_{11}=\left|f_{0}^{\prime}\right|^{2} \frac{1}{\left(1-\left|z_{0}\right|^{2}\right)^{2}}-\left|\frac{f_{0}}{z_{0}}\right|^{4} .
\end{aligned}
$$

Then (1.3) may be written in the following form:

$$
\sum_{i, j=1}^{2} A_{i, j} x_{i} \bar{x}_{j} \geqslant 0
$$

An immediate consequence is

$$
A_{1,1} A_{2,2}-\left|A_{1,2}\right|^{2} \geqslant 0 \text {. }
$$

That is,

$$
\begin{aligned}
\left\{\left|f_{0}^{\prime}\right|^{2} \frac{1}{\left(1-\left|z_{0}\right|^{2}\right)^{2}}-\left|\frac{f_{0}}{z_{0}}\right|^{4}\right\}\left\{\left.\sum_{p, q=1}^{m} \lambda_{p} \bar{\lambda}_{q}|| \frac{f_{p}-f_{q}}{z_{p}-z_{q}}\right|^{2} \frac{1}{\left|1-z_{p} \bar{z}_{q}\right|^{2}}-\left|\frac{f_{p} f_{q}}{z_{p} z_{q}}\right|^{2} \mid\right\} \\
\geqslant\left|\sum_{p=1}^{m} \lambda_{p}\left\{\left|\frac{f_{p}-f_{0}}{z_{p}-z_{0}}\right|^{2} \frac{1}{\left|1-z_{p} \bar{z}_{0}\right|^{2}}-\left|\frac{f_{p}}{z_{p}} \frac{f_{0}}{z_{0}}\right|^{2}\right\}\right|^{2} .
\end{aligned}
$$

Let $\max _{|z|=\rho}|f(z)|=\left|f\left(\rho e^{i \theta_{0}}\right)\right|$ and divide by $\left|f_{0} / z_{0}\right|^{4}$ on both sides of (1.6). Next make $z_{0} \rightarrow e^{i \theta_{0}}$, since 


$$
\left|\frac{z_{0} f_{0}^{\prime}}{f_{0}}\right| \leqslant \frac{1+\left|z_{0}\right|}{1-\left|z_{0}\right|} \text { and } k \leqslant \alpha_{f}=\varlimsup_{\substack{z_{0} \rightarrow e^{i \theta_{0}} \\\left|z_{0}\right|<1}} \frac{\left(1-\left|z_{0}\right|\right)^{2}}{\left|z_{0}\right|}\left|f\left(z_{0}\right)\right|
$$

(1.6) can be reduced to

$$
\begin{aligned}
\sum_{p, q=1}^{m} \lambda_{p} \bar{\lambda}_{q}\left\{\left|\frac{f_{p}-f_{q}}{z_{p}-z_{q}}\right|^{2}\right. & \left.\frac{1}{\left|1-z_{p} \bar{z}_{q}\right|^{2}}-\left|\frac{f_{p} f_{q}}{z_{p} z_{q}}\right|^{2}\right\}\left(\frac{1}{k^{2}}-1\right) \\
& \geqslant \mid \sum_{p=1}^{m} \lambda_{p}\left(\frac{1}{\left|1-z_{p} e^{i \theta_{0}}\right|^{4}}-\left|\frac{f_{p}}{z_{p}}\right|^{2}||^{2} .\right.
\end{aligned}
$$

The method of [2] gives

$$
\begin{aligned}
\left(\frac{1}{k^{2}}-1\right) \sum_{p, q=1}^{m} \lambda_{p} \bar{\lambda}_{q} \int_{0}^{2 \pi} \int_{0}^{2 \pi}\left\{\left|\frac{f\left(\rho_{p} e^{i \theta}\right)-f\left(\rho_{q} e^{i \varphi}\right)}{\rho_{p} e^{i \theta}-\rho_{q} e^{i \varphi}}\right|^{2} \frac{1}{\left|1-\rho_{p} \rho_{q} e^{i(\theta-\varphi)}\right|^{2}}\right. & \left.-\left|\frac{f\left(\rho_{p} e^{i \theta}\right) f\left(\rho_{q} e^{i \theta}\right)}{\rho_{p} \rho_{q}}\right|^{2}\right\} d \theta d \varphi \\
& \geqslant\left|\sum_{p=1}^{m} \lambda_{p} \int_{0}^{2 \pi}\right| \frac{1}{\left|1-\rho_{p} e^{i\left(\theta-\theta_{0}\right)}\right|^{4}}-\left|\frac{f\left(\rho_{p} e^{i \theta}\right)}{\rho_{p}}\right|^{2}|d \theta|^{2} .
\end{aligned}
$$

Inequality (1.8) can be converted to a statement about coefficients by the method of [2]. Thus inequality (1.8) implies inequality (1.2) and Theorem 2 is proved.

THEOREM 3. If $f(z)=z+\sum_{n=2}^{\infty} a_{n} z^{n} \in S(k)$, then

$$
\begin{aligned}
\left(\sum_{K=1}^{2 n} \Lambda_{1}(n, K)\left|a_{K}\right|^{2}\right)^{2}+ & P\left(\sum_{K=1}^{2 n} \Lambda_{1}(n, K)\left(\left|a_{K}\right|^{2}-K^{2}\right)\right)^{2} \\
& \leqslant \sum_{K=1}^{4 n} \Lambda_{2}(n, K)\left|a_{K}\right|^{2},
\end{aligned}
$$

$$
\begin{aligned}
\left(\sum_{K=1}^{4 n} \Lambda_{2}(n, K)\left|a_{K}\right|^{2}\right)^{2} & +P\left\{\sum_{K=1}^{4 n} \Lambda_{2}(n, K)\left(\left|a_{K}\right|^{2}-K^{2}\right)\right\}^{2} \\
& \leqslant \sum_{K=1}^{8 n} \Lambda_{3}(n, K)\left|a_{K}\right|^{2},
\end{aligned}
$$

where $\Lambda_{1}(n, K)=n-|K-n|,|n-K|<n$

$$
\Lambda_{m}(n, K)=\sum_{j=\{(K+1) / 2]}^{2^{m-1} n} \sum_{l=|K-j|}^{j} B(j, l)(l-|j-K|) \Lambda_{m-1}(n, j) \Lambda_{m-1}(n, l)
$$

and $B(j, l)=1, j=l ; 2, j \neq l[1]$. 
The proof of this theorem is similar to [1] except using inequality (1.2) in place of the Fitzgerald inequality.

Proof of Theorem 1. In (1.2) set $L=n, x_{1}=x_{2}=\cdots=x_{n-1}=0$ and $x_{n}=1$ to obtain

$$
\left|a_{n}\right|^{4}+P\left(\left|a_{n}\right|^{2}-n^{2}\right)^{2} \leqslant \sum_{K=1}^{2 n} \Lambda_{1}(n, K)\left|a_{K}\right|^{2} .
$$

From (1.9) and (1.10), it follows that

$$
\begin{aligned}
&\left(\sum_{K=1}^{2 n} \Lambda_{1}(n, K)\left|a_{K}\right|^{2}\right)^{2}+P\left\{\sum_{K=1}^{2 n} \Lambda_{1}(n, K)\left(\left|a_{K}\right|^{2}-K^{2}\right)\right\}^{2} \\
& \leqslant \sum_{K=1}^{4 n} \Lambda_{2}(n, K)\left|a_{K}\right|^{2} \leqslant \sqrt{\sum_{K=1}^{8 n} \Lambda_{3}(n, K)\left|a_{K}\right|^{2}} \leqslant c^{8} n^{8}
\end{aligned}
$$

where $c \doteq 1.0657[1]$.

If $\sum_{K=1}^{2 n} \Lambda_{1}(n, K)\left|a_{K}\right|^{2} \leqslant \sqrt{209 / 140} n^{4}$. Then

$$
\left|a_{n}\right|^{4}+P\left(\left|a_{n}\right|^{2}-n^{2}\right)^{2} \leqslant \sqrt{\frac{209}{140}} n^{4}
$$

by (1.11). It follows that

$$
\left|a_{n}\right| \leqslant n \sqrt{\frac{P+\sqrt{(1+P) \sqrt{209 / 140}-P}}{1+P}}<\left(\frac{209}{140}\right)^{1 / 8} n<c n .
$$

On the other hand, if $\sum_{K=1}^{2 n} \Lambda_{1}(n, K)\left|a_{K}\right|^{2} \geqslant \sqrt{209 / 140} n^{4}$ then, since $\sqrt{209 / 140}$ $>\frac{7}{6}$ and $\Sigma_{K=1}^{2 n} \Lambda_{1}(n, K) K^{2}<\frac{7}{6} n^{4}[2]$

$$
\begin{aligned}
& \left(\sum_{K=1}^{2 n} \Lambda_{1}(n, K)\left|a_{K}\right|^{2}\right)^{2}+P\left(\sqrt{\frac{209}{140}}-\frac{7}{6}\right)^{2} n^{8} \\
& \quad \leqslant\left(\sum_{K=1}^{2 n} \Lambda_{1}(n, K)\left|a_{K}\right|^{2}\right)^{2}+P\left\{\sum_{K=1}^{2 n} \Lambda_{1}(n, K)\left(\left|a_{K}\right|^{2}-K^{2}\right)\right\}^{2} \leqslant c^{8} n^{8}
\end{aligned}
$$

by (1.12).

From (1.11) and (1.15), $c^{8}-p\left(\sqrt{209 / 140}-\frac{7}{6}\right)^{2} \geqslant 0$ and

$$
\begin{aligned}
\left|a_{n}\right|^{4}+P\left(\left|a_{n}\right|^{2}-n^{2}\right)^{2} & \leqslant \sum_{K=1}^{2 n} \Lambda_{1}(n, K)\left|a_{K}\right|^{2} \\
& \leqslant \sqrt{c^{8}-P\left(\sqrt{\frac{209}{140}}-\frac{7}{6}\right)^{2} n^{4}}
\end{aligned}
$$

This gives

$$
\left|a_{n}\right| \leqslant n \sqrt{\frac{P+\sqrt{(1+P)\left\{c^{8}-P(\sqrt{209 / 140}-7 / 6)^{2}\right\}^{1 / 2}-P}}{1+P}}<c n .
$$


This completes the proof of Theorem 1.

\section{Theorem of distortion.}

THEOREM 4. If $f(z)=z+\sum_{n=2}^{\infty} a_{n} z^{n} \in S$, then

$$
\begin{gathered}
|f(z)|^{2} \exp \sum_{n=1}^{\infty} \frac{1}{2 n}\left|G_{n}(z)\right|^{2}+|f(-z)|^{2} \exp \sum_{n=1}^{\infty} \frac{1}{2 n}\left|G_{n}(-z)\right|^{2} \\
\leqslant \frac{|z|^{2}}{(1-|z|)^{4}}+\frac{|z|^{2}}{(1+|z|)^{4}},
\end{gathered}
$$

$$
\begin{gathered}
\left|f^{\prime}(z)\right|^{2} \exp \sum_{n=1}^{\infty} \frac{1}{2 n}\left|G_{n}(z)\right|^{2}+\left|f^{\prime}(-z)\right|^{2} \exp \sum_{n=1}^{\infty} \frac{1}{2 n}\left|G_{n}(-z)\right|^{2} \\
\leqslant\left\{\frac{1+|z|}{(1-|z|)^{3}}\right\}^{2}+\left\{\frac{1-|z|}{(1+|z|)^{3}}\right\}^{2},
\end{gathered}
$$

where $G_{m}(z)=1 / z^{m}+\bar{z}_{m}-F_{m}(1 / f(z))$ and $F_{m}(w)$ is mth order Faber's polynomial.

The proof of Theorem 4 is based on the following inequality [3]:

$$
\sum_{p, q=1}^{m} \lambda_{p} \bar{\lambda}_{q}\left|\frac{f_{p} f_{q}}{z_{p} z_{q}}\right|^{\alpha} \exp \sum_{n=1}^{\infty} \frac{\alpha}{2 n} G_{n}\left(z_{p}\right) \overline{G_{h}\left(z_{q}\right)} \leqslant \sum_{p, q=1}^{m} \lambda_{p} \overline{\lambda_{q}}\left|\frac{f_{p}-f_{q}}{z_{p}-z_{q}}\right|^{\alpha} \frac{1}{\left|1-z_{p} \bar{z}_{q}\right|^{\alpha}}
$$

where $\alpha>0$.

In (2.3), set $m=2, z_{1}=z, z_{2}=-z, \alpha=1$ and $\lambda_{1}=\lambda_{2}=1$ to obtain

$$
\begin{aligned}
\left|\frac{f(z)}{z}\right|^{2} \exp \sum_{n=1}^{\infty} \frac{1}{2 n} \mid & \left.G_{n}(z)\right|^{2}+\left|\frac{f(-z)}{-z}\right|^{2} \exp \sum_{n=1}^{\infty} \frac{1}{2 n}\left|G_{n}(-z)\right|^{2} \\
& +2\left|\frac{f(z) f(-z)}{z^{2}}\right| R \exp \sum_{n=1}^{\infty} \frac{1}{2 n} G_{n}(z) \overline{G_{n}(-z)} \\
& \leqslant \frac{1}{1-|z|^{2}}\left\{\left|f^{\prime}(z)\right|+\left|f^{\prime}(-z)\right|\right\}+\frac{1}{1+|z|^{2}}\left|\frac{f(z)-f(-z)}{z}\right| .
\end{aligned}
$$

Next in (2.3), set $z_{1}=z, z_{2}=-z, \alpha=1$ and $\lambda_{1}=1$, also put $\lambda_{2}=-1$ to obtain

$$
\begin{aligned}
\left|\frac{f(z)}{z}\right|^{2} \exp \sum_{n=1}^{\infty} \frac{1}{2 n}\left|G_{n}(z)\right|^{2}+\left|\frac{f(-z)}{z}\right|^{2} \exp \sum_{n=1}^{\infty} \frac{1}{2 n}\left|G_{n}(-z)\right|^{2} \\
\quad-2\left|\frac{f(z) f(-z)}{z^{2}}\right| R \exp \sum_{n=1}^{\infty} \frac{1}{2 n} G_{n}(z) \overline{G_{n}(-z)} \\
\leqslant \frac{1}{1-|z|^{2}}\left\{\left|f^{\prime}(z)\right|+|f(-z)|\right\}-\frac{1}{1+|z|^{2}}\left|\frac{f(z)-f(-z)}{z}\right| .
\end{aligned}
$$


Adding (2.3), (2.4) we have

$$
\begin{gathered}
\left|\frac{f(z)}{z}\right|^{2} \exp \sum_{n=1}^{\infty} \frac{1}{2 n}\left|G_{n}(z)\right|^{2}+\left|\frac{f(-z)}{-z}\right|^{2} \exp \sum_{n=1}^{\infty} \frac{1}{2 n}\left|G_{n}(-z)\right|^{2} \\
\leqslant \frac{1}{1-|z|^{2}}\left(\left|f^{\prime}(z)\right|+\left|f^{\prime}(-z)\right|\right) .
\end{gathered}
$$

Goluzin proved that [4]

$$
\left|f^{\prime}(z)\right|+\left|f^{\prime}(-z)\right| \leqslant \frac{1+|z|}{(1-|z|)^{3}}+\frac{1-|z|}{(1+|z|)^{3}} .
$$

The inequality (2.1) follows from (2.6) and (2.7). To prove (2.2), we observe that

$$
\left(1-|\zeta|^{2}\right)^{2}\left|\frac{f^{\prime}(\zeta)}{f(\zeta)} \zeta\right|^{2}+\left|\frac{\zeta}{f(\zeta)}\right|^{2} \leqslant(1+|\zeta|)^{4}+(1-|\zeta|)^{4}
$$

This inequality is easily deduced by Goluzin's method [4] and it is omitted here. Inequality (2.8) may be written in the form

$$
\left(1-|\zeta|^{2}\right)^{2}\left|f^{\prime}(\zeta)\right|^{2}+1 \leqslant\left\{(1-|\zeta|)^{4}+(1+|\zeta|)^{4}\right\}\left|\frac{f(\zeta)}{\zeta}\right|^{2} .
$$

In (2.9), set $\zeta=z,-z$ and multiply both sides of (2.6) by $\exp \sum_{n=1}^{\infty}(1 / 2 n)\left|G_{n}(z)\right|^{2}$, $\exp \sum_{n=1}^{\infty}(1 / 2 n)\left|G_{n}(-z)\right|^{2}$ respectively. Adding these results we obtain

$$
\begin{aligned}
\left(1-|z|^{2}\right)^{2} & \left\{\left|f^{\prime}(z)\right|^{2} \exp \sum_{n=1}^{\infty} \frac{1}{2 n}\left|G_{n}(z)\right|^{2}+\left|f^{\prime}(-z)\right|^{2} \exp \sum_{n=1}^{\infty} \frac{1}{2 n}\left|G_{n}(-z)\right|^{2}\right\} \\
& +\exp \sum_{n=1}^{\infty} \frac{1}{2 n}\left|G_{n}(z)\right|^{2}+\exp \sum_{n=1}^{\infty} \frac{1}{2 n}\left|G_{n}(-z)\right|^{2} \\
\leqslant & \left\{(1-|z|)^{4}+(1+|z|)^{4}\right\}\left\{\left|\frac{f(z)}{z}\right|^{2} \exp \sum_{n=1}^{\infty} \frac{1}{2 n}\left|G_{n}(z)\right|^{2}\right. \\
& \left.+\left|\frac{f(-z)}{z}\right|^{2} \exp \sum_{n=1}^{\infty} \frac{1}{2 n}\left|G_{n}(-z)\right|^{2}\right\} \\
\leqslant & \left\{(1-|z|)^{4}+(1+|z|)^{4}\right\}\left\{\frac{1}{(1-|z|)^{4}}+\frac{1}{(1+|z|)^{4}}\right\}
\end{aligned}
$$

by (2.1).

Since $\exp \sum_{n=1}^{\infty}(1 / 2 n)\left|G_{n}(z)\right|^{2}+\exp \sum_{n=1}^{\infty}(1 / 2 n)\left|G_{n}(-z)\right|^{2} \geqslant 2(2.2)$ follows at once from (2.10). Thus the proof of this distortion theorem is complete.

\section{REFERENCES}

1. D. Horowitz, A further refinement for coefficient estimates for univalent functions. Proc. Amer. Math. Soc. 71 (1978), 217-220.

2. C. H. Fitzgerald, Quadratic inequalities and coefficient estimates for schlicht functions. Arch. Rational Mech. Anal. 46 (1972), 356-368.

3. Hu Ke, Jiangxi Shiyuah Xue Bao 2 (1979), 9-16.

4. G. M. Goluzin, Math Sb. 19 (61) 2 (1946), 203-236.

Department of Mathematics, Jiangxi Teacher's College, Nanchang, China 